\title{
Tumores malignos del apéndice cecal en pacientes operados por apendicitis en la IV Región de Chile*
}

\author{
Drs. MARCELO A. BELTRÁN S. ${ }^{1,2}$, RODRIGO TAPIA L. ${ }^{1}$, JUAN MADARIAGA G..$^{1,3}$, \\ RAMÓN I. DÍAZ J. ${ }^{2}$, CRISTÓBAL LARRAÍN T. ${ }^{1,2}$, LUIS JARAMILLO R. ${ }^{1,2}$, \\ FRANCISCO RODRÍGUEZ V. ${ }^{1}$, Int. GABRIEL IRIBARREN R. ${ }^{4}$
}

1 Servicio de Cirugía Hospital San Juan de Dios de La Serena.

2 Facultad de Medicina Universidad Católica del Norte.

3 Unidad de Anatomía Patológica Hospital San Pablo de Coquimbo.

4 Interno Facultad de Medicina Universidad Católica del Norte. IV Región, Chile.

\section{Abstract}

\section{Malignant appendicular tumors on patients operated for acute appendicitis}

Background: The most common malignant appendicular neoplasm is the neuroendocrine welldifferentiated tumor (NET). Other malignant tumors are uncommon, reported with a frequency of $0.2 \%$ of all appendectomies. Aim: To describe the characteristics of appendiceal malignant tumors found in regional hospitals of Northern Chile. Material and Methods: All patients operated for acute appendicitis between 2005 and 2011, in which a malignant appendiceal tumor was found in the pathological study, were analyzed. Clinical and survival information was obtained from medical records and national death registries. Results: We analyzed 8.972 appendectomy records. Appendicular tumors were found in 40 patients (0.4\%). Thirty patients $(0.3 \%)$ had malignant tumors. NET was the most common tumor found in 19 patients $(63 \%)$. The tumor was smaller than $1 \mathrm{~cm}$ in 16 cases (53\%). In 5 patients (17\%), the tumor was larger than $2 \mathrm{~cm}$ with the appendicular base infiltrated by malignant cells. Most patients (83\%) presented with stage I tumors. In 23 patients (77\%), the appendectomy was considered sufficient and definitive treatment. Conclusions: In this series of patients, appendicular tumors had similar characteristics to those reported abroad. All patients had a favorable evolution without associated mortality or tumor relapse.

Key words: Appendix, malignant tumor, appendectomy.

\section{Resumen}

Introducción: El tumor apendicular maligno encontrado con mayor frecuencia es el tumor neuroendocrino bien diferenciado (NET). Otros tumores malignos se reportan con una frecuencia menor a $0,2 \%$ de todas

"Parte de este estudio fue presentado en el LXXXV Congreso Chileno e Internacional de Cirugía en la ciudad de La Serena, noviembre de 2012".

*Recibido el 12 de junio de 2013 y aceptado para publicación el 24 de junio de 2013.

Los autores no refieren conflictos de interés.

Correspondencia: Dr. Marcelo A. Beltrán S.

Casilla 912. La Serena, Chile.

beltran_01@yahoo.com 
las apendicectomías. El objetivo del presente estudio es describir las características de los tumores malignos del apéndice en la IV Región de Chile. Material y Método: Se estudió una cohorte histórica constituida por pacientes operados en los hospitales de La Serena, Coquimbo y Ovalle entre enero de 2005 y diciembre de 2011. Los resultados se reportan mediante estadística descriptiva. Resultados: Se estudiaron 8.972 apendicectomías. En 40 pacientes $(0,4 \%)$ se diagnosticaron tumores apendiculares de los cuales $30(0,3 \%)$ fueron malignos y fueron incluidos en este análisis. El tipo histológico maligno más frecuente fue el NET en 19 casos (63\%). En 16 casos (53\%) el tumor fue menor a $1 \mathrm{~cm}$. En 5 casos (17\%) el tumor fue mayor a $2 \mathrm{~cm}$ con compromiso de la base. La mayoría de los pacientes $(83 \%)$ se presentaron con tumores en estadio I y los demás con tumores en estadio II. En 23 pacientes (77\%) la apendicectomía fue considerada como el tratamiento definitivo. Conclusiones: Las características generales de los tumores malignos del apéndice en los pacientes operados por apendicitis en la IV Región de Chile son similares a las características reportadas en series nacionales e internacionales. En la presente cohorte, los pacientes tuvieron una evolución favorable sin mortalidad asociada al tumor y sin recurrencias conocidas hasta la fecha actual.

Palabras clave: Apendicitis, apendicectomía, tumores apendiculares.

\section{Introducción}

Los tumores apendiculares tienen una baja incidencia que varía aproximadamente entre $0,2 \%$ a $0,5 \%$ de todas las neoplasias gastrointestinales, encontrándose en una frecuencia menor a $2 \%$ de todos los pacientes sometidos a apendicectomía de urgencia por apendicitis ${ }^{1-5}$. La presentación habitual de la mayoría de estos tumores es el proceso inflamatorio agudo del apéndice, razón por la cual el diagnóstico definitivo se conoce sólo con el informe histopatológico ${ }^{1-4}$. En algunos casos excepcionales, el diagnóstico de un tumor apendicular se sospecha durante la cirugía o en el período preoperatorio, planteando un problema respecto al tratamiento quirúrgico apropiado en esta circunstancia ${ }^{1,3,4}$. Los tumores apendiculares pueden ser benignos, tales como el cistoadenoma mucinoso asociado a otros tumores del ovario o colon, el mucocele simple, los neuromas y neurofibromas en pacientes con neurofibromatosis tipo I y los tumores estromales ${ }^{1,6}$. El tumor maligno encontrado con mayor frecuencia, hasta en $50 \%$ a $70 \%$ de todos los casos, es el tumor neuroendocrino bien diferenciado (NET), previamente conocido como tumor carcinoide, con una incidencia que varía entre $0,1 \%$ a $1,5 \%$ y una prevalencia de $0,32 \%{ }^{1-7}$. Aproximadamente $19 \%$ de todos los NET gastrointestinales se localizan en el apéndice y constituyen entre $32 \%$ y $57 \%$ de todas las neoplasias apendiculares ${ }^{7-13}$. Otros tumores epiteliales primarios como el adenocarcinoma y el cistoadenocarcinoma mucinoso del apéndice son aún más infrecuentes y se reportan con una frecuencia menor a $0,2 \%$ de todas las apendicectomías ${ }^{1,3,4,6,14}$, constituyendo entre $2 \%$ y $18 \%$ de todas las neoplasias apendiculares ${ }^{3,4,14}$. El desarrollo de una base de datos confiable durante los últimos años en la IV Región de Chile y la necesidad de conocer la realidad regional respecto a una patología tan infrecuente fueron las razones que nos llevaron a desarrollar el presente estudio. El objetivo es describir las características de los tumores malignos del apéndice en nuestra región, la experiencia con el tratamiento y la sobrevivencia de estos pacientes.

\section{Material y Método}

El presente es un estudio retrospectivo y descriptivo de una cohorte histórica constituida por pacientes operados por tumores apendiculares malignos en los hospitales San Juan de Dios de La Serena, San Pablo de Coquimbo y Antonio Tirado Lanas de Ovalle durante un período de 7 años comprendido entre enero de 2005 y diciembre de 2011. En estos hospitales se concentra la actividad quirúrgica de la IV Región por lo que el universo de pacientes es representativo de la región ${ }^{15}$. Se incluyeron todos los pacientes operados por apendicitis con informe histopatológico que confirmara el diagnóstico de tumor apendicular maligno. Se excluyeron pacientes en quienes la apendicectomía fue incidental, pacientes con tumores apendiculares benignos confirmados por patología y aquellos pacientes en los que el informe histopatológico no se encontrara disponible o no existiera. Se estudiaron variables demográficas, clínicas, quirúrgicas, histológicas y seguimiento de los pacientes mediante revisión de fichas clínicas, control en policlínico de cirugía y registros del Registro Civil. El análisis se realizó mediante estadística descriptiva de las variables categóricas, dicotómicas y continuas.

\section{Definiciones}

Los NET son tumores con diferenciación neuroendocrina, se dividen en 4 subtipos histológicos: tumores de células espumosas, tumores de células compuestas, tumores de células enterocromafines y tumores de células atípicas. Se utilizó la clasificación TNM propuesta por Landry para tumores NET apendiculares ${ }^{11}$. Los adenocarcinomas se definieron 
como la neoplasia epitelial maligna del apéndice que invade más allá de la muscular de la mucosa, estos tumores pueden ser de tres tipos histológicos: adenocarcinoma tubular, adenocarcinoma mucinoso y carcinoma con células en anillo de sello. Esta designación en tres tipos es sólo descriptiva debido a que no constituyen condiciones patológicas diferentes. Debido a que en nuestros pacientes sólo se encontraron tumores epiteliales, en este reporte se utilizó la clasificación histológica de la World Health Organization para tumores epiteliales del apéndice que divide a estas neoplasias en adenomas, carcinomas, tumores neuroendocrinos y otros más infrecuentes como los carcinoides tubulares, carcinoides mucinosos, etc. La Etapificación de los adenocarcinomas se realizó mediante la clasificación TNM para tumores malignos epiteliales del apéndice que coincide con la clasificación propuesta por Landry ${ }^{11}$. Debido a que en el sistema público regional no existe la posibilidad de medir el Ki67, el grado de diferenciación tumoral fue medido mediante la visualización del número de mitosis por 10 campos de aumento mayor calculando el grado de inmadurez celular y se clasificaron de acuerdo a la American Joint Commission on Cancer, en los siguientes grados: Grado $\mathrm{X}$, el grado no puede evaluarse; Grado 1, bien diferenciado con no más de $25 \%$ de las células inmaduras; Grado 2, moderadamente diferenciado con $25 \%$ a $50 \%$ de las células inmaduras; Grado 3, pobremente diferenciado con $50 \%$ a $75 \%$ de las células inmaduras; y Grado 4, indiferenciado cuando más del $75 \%$ de las células son inmaduras.

\section{Resultados}

En el período de estudio, se realizaron 9.245 apendicectomías $(100 \%)$. Fueron excluidos 273 casos (3\%) debido a que se trató de apendicectomías incidentales (140 casos) o no se encontró el informe de biopsia (133 casos). Se incluyeron en el protocolo 8.972 apendicectomías $(97 \%)$ por apendicitis. En 40 pacientes $(0,4 \%)$ se diagnosticaron tumores apendiculares, de los cuales 30 $(0,3 \%)$ fueron malignos y fueron incluidos en este análisis (Figura 1). La distribución de estos pacientes por hospital, las características demográficas y el tipo de tratamiento se detallan en la Tabla 1. La mayoría de los tumores malignos se operaron en el Hospi- tal de Ovalle, la mayoría de los pacientes fueron de género femenino, en todos ellos el procedimiento quirúrgico inicial fue la apendicectomía y en 27 pacientes $(90 \%)$ con tumores malignos se encontró apendicitis aguda asociada.

\section{Histopatología}

El tipo histológico maligno más frecuente fue el NET en 19 casos (63\%). La ubicación del tumor en el apéndice fue distal en 16 casos (53\%) y difuso en 3 casos $(10 \%), 2$ casos de NET y un adenocarcinoma tubular. En 16 casos (53\%) el tumor fue menor a $1 \mathrm{~cm}$. En 5 casos (17\%) el tumor fue mayor a $2 \mathrm{~cm}$ con compromiso de la base. En 12 casos (40\%) el tumor comprometía la subserosa y en 11 casos (37\%) el compromiso tumoral incluía la muscular propia. En un paciente con adenocarcinoma tubular el tumor que comprometía la base y medía más de $2 \mathrm{~cm}$ estaba perforado. En 23 casos $(77 \%)$ el tumor no infiltraba el mesoapéndice y en ningún caso se encontraron linfonodos metastásicos. Todos los NET fueron tumores bien diferenciados (Grado 1). En 24 casos (80\%) no se observó permeación linfovascular. El detalle de los hallazgos histopatológicos se encuentra en la Tabla 2.

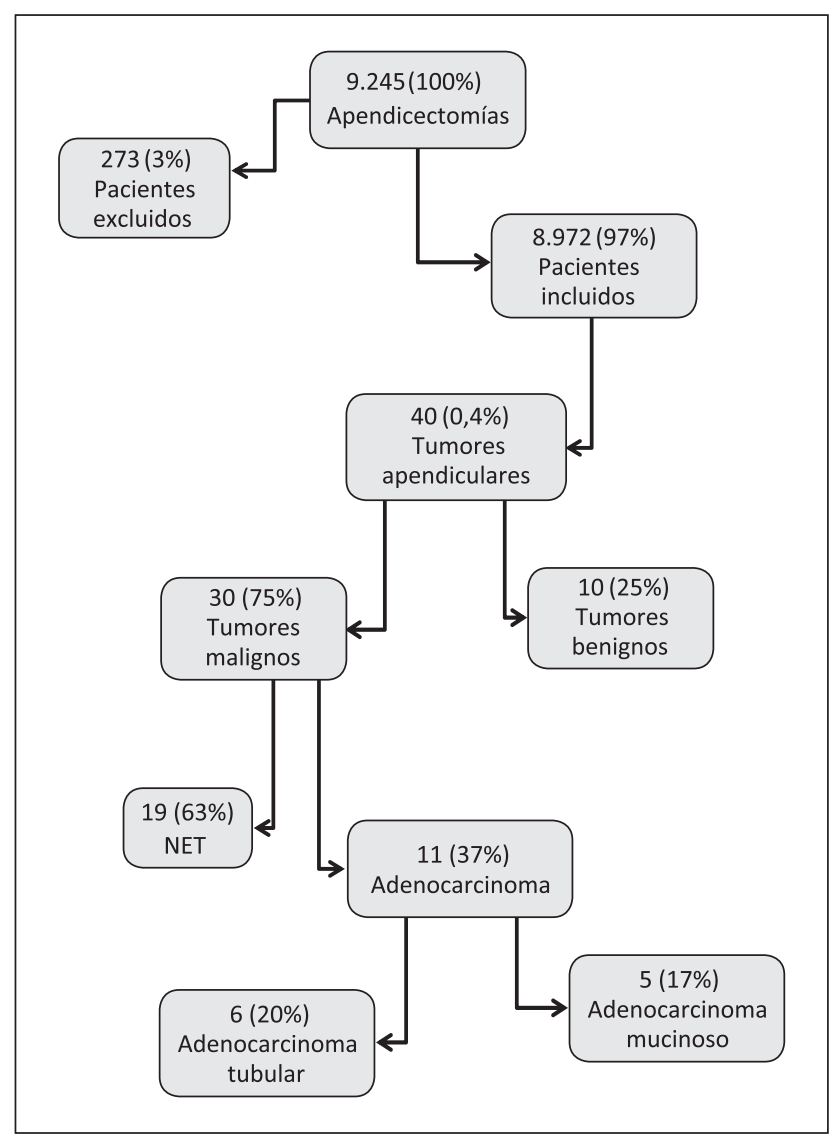

Figura 1. Flujo de pacientes con tumores apendiculares malignos incluidos en el estudio. 
Tabla 1. Características generales

\begin{tabular}{|lccc|}
\hline Variable & Tumores malignos & Tumores benignos & Total \\
Hospital de Ovalle & 14 & 2 & 16 \\
Hospital de Coquimbo & 10 & 7 & 17 \\
Hospital de La Serena & 6 & 1 & 7 \\
Edad (años) & $33,2 \pm 22,4(9-80)$ & $35,4 \pm 19(21-66)$ & $35,1 \pm 17(9-80)$ \\
Género (M/F) & $9(30 \%) / 21(70 \%)$ & $1(10 \%) / 9(90 \%)$ & $10(25 \%) / 30(75 \%)$ \\
Presentación Clínica (Apendicitis) & $30(100 \%)$ & $10(100 \%)$ & $40(100 \%)$ \\
Cirugía Inicial (Apendicectomía abierta) & $30(100 \%)$ & $10(100 \%)$ & $40(100 \%)$ \\
Apendicitis Aguda Asociada & $27(90 \%)$ & $8(80 \%)$ & $35(87,5 \%)$ \\
\hline
\end{tabular}

Tabla 2. Características histopatológicas de 30 tumores apendiculares malignos

\begin{tabular}{|c|c|c|}
\hline Variable & Característica & \\
\hline Tipo histológico & $\begin{array}{l}\text { NET } \\
\text { Adenocarcinoma tubular } \\
\text { Cistoadenocarcinoma mucinoso }\end{array}$ & $\begin{aligned} 19 & (63 \%) \\
6 & (20 \%) \\
5 & (17 \%)\end{aligned}$ \\
\hline Ubicación en el apéndice & $\begin{array}{l}\text { Distal o Apex } \\
\text { Medio } \\
\text { Proximal } \\
\text { Difuso }\end{array}$ & $\begin{aligned} 16 & (53 \%) \\
6 & (20 \%) \\
5 & (17 \%) \\
3 & (10 \%)\end{aligned}$ \\
\hline Tamaño $(\mathrm{cm})$ & $\begin{array}{l}<1 \\
1-2 \\
>2\end{array}$ & $\begin{aligned} 16 & (53 \%) \\
9 & (30 \%) \\
5 & (17 \%)\end{aligned}$ \\
\hline Compromiso parietal & $\begin{array}{l}\text { Mucosa } \\
\text { Submucosa } \\
\text { Muscular Propia } \\
\text { Subserosa } \\
\text { Serosa }\end{array}$ & $\begin{array}{rr}1 & (3 \%) \\
3 & (10 \%) \\
11 & (37 \%) \\
12 & (40 \%) \\
3 & (10 \%)\end{array}$ \\
\hline Compromiso del mesoapéndice & $\begin{array}{l}\text { Sí } \\
\text { No }\end{array}$ & $\begin{array}{rr}7 & (23 \%) \\
23 & (77 \%)\end{array}$ \\
\hline Compromiso linfonodal & $\begin{array}{l}\text { Sí } \\
\text { No }\end{array}$ & $30(100 \%)$ \\
\hline Diferenciación & $\begin{array}{l}\text { Bien diferenciado (Grado 1) } \\
\text { Moderadamente diferenciado (Grado 2) } \\
\text { Pobremente diferenciado (Grado 3) }\end{array}$ & $\begin{array}{rr}25 & (83 \%) \\
4 & (14 \%) \\
1 & (3 \%)\end{array}$ \\
\hline Permeación linfovascular & $\begin{array}{l}\text { Sí } \\
\text { No }\end{array}$ & $\begin{array}{rr}6 & (20 \%) \\
24 & (80 \%)\end{array}$ \\
\hline
\end{tabular}

\section{Tratamiento}

La mayoría de los pacientes (83\%) se presentaron con tumores en estadio I y los demás con tumores en estadio II (Tabla 3). En 23 pacientes (77\%) la apendicectomía fue considerada como el tratamiento definitivo. En 7 pacientes se consideró necesaria una posible reintervención. Una paciente con un NET difuso y mayor a $2 \mathrm{~cm}$ con compromiso de la base y la subserosa, con infiltración del mesoapéndice y permeaciones linfáticas y perineurales, fue sometida a una hemicolectomía derecha la cual no demostró la presencia alguna de neoplasia y la disección linfática de 38 linfonodos fue negativa para metástasis. Un segundo caso fue el de un paciente con un cistoadenocarcinoma mucinoso difuso mayor a $2 \mathrm{~cm}$, bien diferenciado, con compromiso de la base y la subserosa, sin permeaciones linfáticas o perineurales; se realizó una hemicolectomía derecha en la que no se 
Tabla 3. TNM

\begin{tabular}{|c|c|c|c|}
\hline & NET & Adenocarcinoma & Total \\
\hline $\mathrm{T} 1$ & $17(56 \%)$ & $8 \quad(27 \%)$ & $25 \quad(83 \%)$ \\
\hline $\mathrm{T} 2$ & $2(6 \%)$ & $3(10 \%)$ & $5 \quad(17 \%)$ \\
\hline No & $19(63 \%)$ & $11 \quad(37 \%)$ & $30(100 \%)$ \\
\hline N1 & - & - & - \\
\hline M0 & - & - & - \\
\hline M1 & - & - & - \\
\hline Estadio I & $17(56 \%)$ & $8 \quad(27 \%)$ & $25 \quad(83 \%)$ \\
\hline Estadio II & $2(6 \%)$ & $3(37 \%)$ & $5(17 \%)$ \\
\hline
\end{tabular}

demostró presencia de neoplasia y tampoco metástasis linfáticas en 35 linfonodos. Un tercer caso fue el de una paciente con un cistoadenocarcinoma mucinoso de la punta que medía $2,5 \mathrm{~cm}$ en la cual se realizó una hemicolectomía derecha que no evidenció presencia tumoral, además de 30 linfonodos negativos. Un cuarto caso fue el de una paciente de 77 años con un adenocarcinoma tubular pobremente diferenciado en la cual la hemicolectomía no demostró tumor y 39 linfonodos fueron negativos para metástasis. Finalmente el quinto caso fue el de una paciente de 40 años con un NET pobremente diferenciado de 2,1 $\mathrm{cm}$ en la que la hemicolectomía y la disección de 34 linfonodos fueron negativas para metástasis tumorales. Dos pacientes con criterios de reintervención no fueron operados. El primero con un NET del ápex apendicular con permeaciones linfáticas fue derivado por la Unidad de Cirugía Infantil a otro centro. En el segundo paciente con un adenocarcinoma tubular moderadamente diferenciado del ápex apendicular y compromiso de la serosa, se consideró que la apendicectomía fue suficiente debido a las comorbilidades presentes y al riesgo quirúrgico.

\section{Seguimiento}

La cohorte fue seguida entre 1 y 7 años (promedio 4,5 años), en 10 pacientes la sobrevivencia fue superior a 5 años, en 7 pacientes fue superior a 3 años y en 9 pacientes superior a un año. Un paciente con un NET falleció a los 72 años de edad, después de 6 años de la operación por causas cardiovasculares. Tres pacientes fueron perdidos al seguimiento. Ningún paciente falleció por causas asociadas a los tumores apendiculares.

\section{Discusión}

La incidencia de tumores apendiculares en los pacientes de la IV Región de Chile es similar a la reportada en otras series nacionales, en las que se menciona una cifra que varía entre $0,07 \mathrm{y}$ $0,8 \% \%^{2,8,13,16}$. En estudios extranjeros se encuentra una incidencia que varía entre $0,2 \%$ y $0,95 \%^{1,3,4,7}$. La forma de presentación habitual es la apendicitis aguda asociada a los tumores apendiculares que se presenta con una frecuencia que varía entre $90 \%$ y $100 \%$ de los casos ${ }^{1-5,10,12-14}$; en esta cohorte, todos los pacientes se presentaron clínicamente como apendicitis aguda. Así como se encuentra extensamente descrito ${ }^{2-4,7-13,16}$, el tumor más frecuentemente encontrado fue el NET, que en esta serie constituye el $63 \%$ de los tumores encontrados. Otros estudios han reportado la presencia de NET en $32 \%$ a $73 \%$ de los pacientes con tumores apendiculares ${ }^{1-4,16}$. Los NET se presentan con una mayor frecuencia en pacientes femeninos con un promedio de edad que se sitúa entre la tercera y cuarta década de la vida ${ }^{7-9,11}$. Los adenocarcinomas son más comunes en pacientes masculinos y se presentan alrededor de la quinta o sexta década de la vida y con una frecuencia de $3,2 \%$ a $24 \%{ }^{1-3,14,16}$; estas características fueron compartidas por los pacientes de este reporte y coinciden con las publicaciones nacionales e internacionales ${ }^{1-4,16}$.

Respecto a la ubicación del tumor, el tercio distal del apéndice se encontraba comprometido en la mayoría de los casos, tal y como ha sido suficientemente reportado ${ }^{2-4,10}$. En la presente cohorte, así como en otras publicaciones, la mayoría de los tumores apendiculares se localizaron en el ápex apendicular ${ }^{3,7,10}$, razón a la cual, en conjunto con la presentación precoz debido a la inflamación aguda del apéndice, se atribuye su buen pronóstico general. Habitualmente la cirugía inicial en estos casos es la apendicectomía debido a que la presentación clínica más común de los tumores apendiculares malignos es la apendicitis ${ }^{3,4,10,12,13,16}$. Todos los pacientes de esta cohorte se presentaron como apendicitis aguda y como consecuencia la cirugía inicial fue una apendicectomía abierta. La apendicectomía laparoscópica no se asocia con un peor pronóstico en los pacientes operados por apendicitis asociada a tumores apendiculares comparada con la apendicectomía abierta ${ }^{5}$.

En general los tumores malignos del apéndice se comportan de forma poco agresiva y son de buen pronóstico ${ }^{2,3}$, hechos confirmados por los hallazgos en esta cohorte, en la que ningún tumor se encontraba en un estadio avanzado. Cuando se tomó la decisión de someter al paciente a una hemicolectomía derecha formal de acuerdo a las indicaciones habituales, no se encontraron tumores avanzados, metástasis linfáticas o metástasis a distancia y ningún paciente falleció por el tumor apendicular. Recientemente se ha cuestionado la necesidad de realizar una hemicolectomía derecha en pacientes con NET ${ }^{2,3,7,9,10}$. La base de este cuestionamiento se apoya en estudios que han demostrado que después de una apendicectomía con hallazgo incidental de un NET e independientemente del tamaño del tumor 
o de la cirugía practicada ningún paciente falleció debido al tumor ${ }^{2,3,7,9}$. La serie más importante publicada sobre $\mathrm{NET}^{7}$, estableció que en un período superior a 28 años de seguimiento sólo 1 paciente con un NET mayor a $2 \mathrm{~cm}$ desarrolló recurrencia regional y metástasis, los mismos autores recomendaron la apendicectomía como tratamiento único en tumores menores a $2 \mathrm{~cm}$; en tumores mayores a 2 $\mathrm{cm}$ recomendaron la hemicolectomía derecha pero sólo en pacientes jóvenes con bajo riesgo quirúrgico y en pacientes con invasión vascular o compromiso del meso apendicular. El reciente desarrollo de un sistema TNM para tumores NET demostró que tumores en estadios I y II no requieren otro tratamiento quirúrgico como la resección del colon derecho ${ }^{11}$. En esta serie los pacientes que fueron considerados para hemicolectomía derecha, además de tener tumores mayores a $2 \mathrm{~cm}(\mathrm{~T} 2)$, presentaron invasión vascular en 6 casos y compromiso del mesoapéndice en 7 casos.

Así también se encuentra en la polémica el tratamiento con hemicolectomía derecha para los adenocarcinomas apendiculares ${ }^{2,8}$, sin embargo, la evidencia actual confirma la necesidad de realizar una hemicolectomía derecha en pacientes con adenocarcinoma apendicular aun cuando sean menores a 2 $\mathrm{cm}^{2-4,14,16}$. En esta cohorte la mayoría de los pacientes tenían un NET y a diferencia de la experiencia de otros autores ${ }^{2,7}$, ningún paciente falleció debido al tumor apendicular y sólo en cinco casos se realizó una hemicolectomía derecha sin encontrarse un tumor avanzado. La supervivencia prolongada de pacientes con tumores apendiculares depende fundamentalmente del tipo histológico del tumor ${ }^{2}$. Consecuentemente y debido a que en esta serie ningún paciente falleció debido a su tumor apendicular, no es posible evaluar la eficacia de la hemicolectomía derecha en el tratamiento de los tumores apendiculares malignos en los pacientes de la IV Región.

\section{Conclusiones}

Las características generales de los tumores malignos del apéndice cecal en los pacientes operados por apendicitis en la IV Región de Chile son similares a las características reportadas en series nacionales y a lo descrito en las publicaciones internacionales. En la presente cohorte, los pacientes operados con tumores apendiculares tuvieron una evolución favorable sin mortalidad asociada al tumor y sin recurrencias hasta la fecha actual.

\section{Referencias}

1. Esmer-Sánchez DD, Martínez-Ordaz JL, Román-Zepeda P, Sánchez-Fernández P, Medina-González E. Tumores apendiculares: Revisión clínico-patológica de 5.307 apendicectomías. Cir Cir. 2004;72:375-8.

2. Butte JM, García MA, Torres J, Salinas M, Duarte I, Pinedo G, y cols. Tumores del apéndice cecal: Análisis anatomoclínico y evaluación de la sobrevida alejada. Rev Chil Cir. 2007;59:217-22.

3. Castañeda LE, Valanci S, Belmonte C. Incidencia de tumores del apéndice en el Centro Médico ABC. An Med. 2009;54:201-5.

4. Lee WS, Choi ST, Lee JN, Kim KK, Park YH, Baek JH. A retrospective clinicopathological analysis of appendiceal tumors from 3,744 appendectomies: A singleinstitution study. Int J Colorectal Dis. 2011;26:617-21.

5. Bucher P, Mathe Z, Demirag A, Morel Ph. Appendix tumors in the era of laparoscopic appendectomy. Surg Endosc. 2004;18:1063-6.

6. Ruiz-Tovar J, Teruel DG, Castiñeiras VM, Dehesa AS, Quindós PL, Molina EM. Mucocele of the appendix. World J Surg. 2007;31:542-8.

7. Moertel CG, Weiland LH, Nagorney DM, Dockerty MB. Carcinoid tumor of the appendix: Treatment and prognosis. N Engl J Med. 1987;317:1699-701.

8. Uherek FP, Barría CA, Larraín CT, Birrer EG. Carcinoide apendicular: Comunicación de 6 casos y actualización del tema. Cuad Cir. 2004;18:52-6.

9. Bamboat ZM, Berger DL. Is right hemicolectomy for 2.0-cm appendiceal carcinoids justified? Arch Surg. 2006;141:349-52.

10. Tchana-Sato V, Detry O, Polus M, Thiry A, Detroz B, Maweja S, et al. Carcinoid tumor of the appendix: A consecutive series from 1237 appendectomies. World J Gastroenterol. 2006;12:6699-701.

11. Landry CS, Woodall C, Scoggins CR, McMasters KM, Martin RC. Analysis of 900 appendiceal carcinoid tumors for a proposed predictive staging system. Arch Surg. 2008;143:664-70.

12. in't Hof KH, van der Wal HC, Kazemier G, Lange JF. Carcinoid tumor of the appendix: An analysis of 1485 consecutive emergency appendectomies. J Gastrointest Surg. 2008;12:1436-8.

13. Buchholtz M, Zúñiga JM, Valdés F, Fernández R, Neveu $\mathrm{R}$, Trujillo C, y cols. Carcinoide gastrointestinal: Experiencia de 7 años en el Instituto Nacional del Cáncer (2000-2006). Rev Chil Cir. 2010;62:480-5.

14. Nitecki SS, Wolff BG, Schlinkert R, Sarr MG. The natural history of surgically treated primary adenocarcinoma of the appendix. Ann Surg. 1994;219:51-7.

15. Beltrán MA, Vicencio AO, Barra MM, Contreras MA, Wilson CS, Cruces KS. Resultados del tratamiento quirúrgico de los tumores del estroma gastrointestinal (GIST) en la IV Región de Chile. Rev Chil Cir. 2011;63:290-6.

16. Tapia O, Manterola C, Villaseca M, Araya JC, Guzmán $\mathrm{P}$, Roa JC. Descripción clínico-morfológica y factores pronósticos en carcinomas del apéndice cecal: Estudio de cohorte. Rev Chil Cir. 2010; 62:255-61. 